\title{
Structural, Magnetic and Electrical Properties of Cu-Mg Ferrites
}

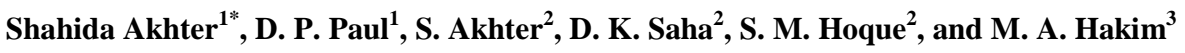 \\ ${ }^{1}$ Department of Physics, University of Chittagong, Chittagong-4331, Bangladesh \\ ${ }^{2}$ Materials Science Division, Atomic Energy Centre, Dhaka-1000, Bangladesh \\ ${ }^{3}$ Dept. of Glass and Ceramic Engineering, Bangladesh University of Engineering and Technology, \\ Dhaka, Bangladesh
}

Received 17 December 2013, accepted in final revised form 22 April 2014

\begin{abstract}
$\mathrm{Cu}_{1-\mathrm{x}} \mathrm{Mg}_{\mathrm{x}} \mathrm{Fe}_{2} \mathrm{O}_{4}$ ferrites with $(x=0.2,0.4,0.6,0.8$ and 1.0) has been synthesized by double sintering ceramic technique at $1150^{\circ} \mathrm{C}$ for $2 \mathrm{hr}$ and investigated for their structure, microstructure, complex permeability behaviour, DC and AC resistivity, dielectric constant measurements. X-ray analysis indicated the formation of single-phase cubic spinel structure for all samples and the lattice parameter was found to decrease with increasing $\mathrm{Mg}$ content. Grain size was found to decrease from 34.36 to $4.16 \mu \mathrm{m}$ with the increase of $\mathrm{Mg}$ contents. The real part of initial permeability $\left(\mu^{\prime}\right)$ remained constant up to certain lower range of frequency after it decreased slightly to lower value of permeability at which imaginary part of permeability $\left(\mu^{\prime \prime}\right)$ showed a maximum value. The decrease of $\mu^{\prime}$ is observed with the increase of $\mathrm{Mg}$ content which is attributed to a decrease of grain size. DC electrical resistivity and dielectric constant decreased with increasing $\mathrm{Mg}$ content and AC resistivity decreased with increasing frequency, exhibiting normal ferrimagnetic behaviour. The observed variation of electrical and dielectric properties has been explained on the basis of the electronic hopping frequency between $\mathrm{Fe}^{2+}$ and $\mathrm{Fe}^{3+}$ ions in present samples.
\end{abstract}

Keywords: Ferrites; XRD; Micrographs; Complex permeability; DC resistivity.

(ㅇ 2014 JSR Publications. ISSN: 2070-0237 (Print); 2070-0245 (Online). All rights reserved.

doi: http://dx.doi.org/10.3329/jsr.v6i2.17351 J. Sci. Res. 6 (2), 205-215 (2014)

\section{Introduction}

Spinel ferrites are important metal oxides that posses ferrimagnetic and are widely applied in electrical devices, such as information storage and microwave devices, because of their excellent electromagnetic properties, high electrical resistivity and low eddy current $[1,2]$. The high electrical resistivity and good magnetic properties make the ferrites an excellent core material for power transformers in electronic and

\footnotetext{
*Corresponding author: shahida212@yahoo.com
} 
telecommunication applications. These ferrites possessing cubic close-packed structure of oxygen ions are described by the formula $(\mathrm{A})[\mathrm{B}]_{2} \mathrm{O}_{4}$ where $(\mathrm{A})$ and $[\mathrm{B}]$ represent tetrahedral and octahedral sites respectively. The site occupancy is often depicted in the chemical formula as $\left(\mathrm{M}_{1-\delta} \mathrm{Fe}_{\delta}\right)\left[\mathrm{M}_{\delta} \mathrm{Fe}_{2-\delta}\right] \mathrm{O}_{4}$ where round and square brackets denote the Aand $\mathrm{B}$-sites, respectively, $\mathrm{M}$ represents a metal cation and $\delta$ for spinel ferrites is defined as the fraction of tetrahedral (A)-sites occupied by trivalent cations. Accordingly, for a normal spinel $\delta=0$ and for a completely inverse spinel $\delta=1$. The magnetic and the electric properties of such a ferrite system depend upon the type of metal cations and their distribution among the two interstitial sites that is A- and B-sites. The electrical properties of ferrites also markedly depend on the sintering time and temperature, chemical composition, preparation conditions, and the quality and type of additives. Among all ferrites, Copper ferrite $\left(\mathrm{CuFe}_{2} \mathrm{O}_{4}\right)$ is one of the most important ferrites which has both magnetic and semiconducting properties and have widely used in electronic industry [3]. Recently there is a growing interest on $\mathrm{Mg}$-based ferrites to use in transformer, ferro-fluids, magnetic cores of coils, catalysts, gas sensors and humidity sensors because of their high resistivity and low dielectric lossess [4-6]. Extensive works has been reported regarding the non-magnetic substitution in the different ferrites system in order to improve their magnetic and electrical properties. The aim of this present work is twofold: First the phase analysis and microstructural study by XRD and SEM. Second to observe the magnetic behaviour by complex permeability, electrical and dielectric properties of the non-magnetic $\mathrm{Mg}$ substituted in $\mathrm{Cu}-\mathrm{Mg}$ ferrites.

\section{Experimental}

\subsection{Sample Preparation}

$\mathrm{Cu}_{1-\mathrm{x}} \mathrm{Mg}_{\mathrm{x}} \mathrm{Fe}_{2} \mathrm{O}_{4}(x=0.2,0.4,0.6,0.8,1.0)$ has been prepared by the double sintering ceramic technique. High purity reagent nano-powders of $\mathrm{CuO}, \mathrm{MgO}$, and $\mathrm{Fe}_{2} \mathrm{O}_{3}$ were mixed according to their molecular weight. Intimate mixing for the materials was carried out for 4 hours using agate mortar and then the materials in ethyl alcohol were poured into an agate jar placed into a planetary grinding miller, and then the materials were continuously milled for 4 hours. The slurry was dried and was pressed into disc shape sample. The disc shaped sample was pre-sintered at $1000^{\circ} \mathrm{C}$ for 4 hours at a heating rate of $4^{0} \mathrm{C} /$ minute in air to form ferrite through chemical reaction. The pre-sintered material was again crushed and wet milled for another 4 hours in distilled water to reduce it to small crystallites of uniform size. The mixture was dried and a few drops of saturated solution of polyvinyl alcohol were added as a binder. The resulting powders were pressed uniaxially under a pressure of $\left(15-20 \mathrm{kN} / \mathrm{cm}^{2}\right)$ in a stainless steel dies to make pellets. The pressed pellets ( $9 \mathrm{~mm}$ diameter, $3 \mathrm{~mm}$ thickness) toroids $(12 \mathrm{~mm}$ outer diameter, 8 
$\mathrm{mm}$ inner diameter and $4 \mathrm{~mm}$ thickness) were then finally sintered at $1150^{\circ} \mathrm{C} 2$ hours. All samples were heated slowly in the programmable Muffle furnace (Model HTL 10/17, Germany) at the rate of about $220^{\circ}$ /hours increase to avoid cracking of the samples.

\subsection{Sample characterization}

Phase analysis was done by X-ray diffraction using Phillips (PW3040) X' Pert PRO Xray diffractometer. The microstructures of the samples were done by a scanning electron microscope (SEM) (model: FEI Inspect S50). The SEM micrographs were taken on the smooth surface of the pellet-shaped polished samples. Before taking micrographs, the surface of the samples were thermally etched at a temperature of $150^{\circ} \mathrm{C}$ below the sintering temperature. Frequency dependence complex permeability of the toroid shaped samples at room temperature was measured with 6500B Impedance Analyzer at frequency range from $1 \mathrm{kHz}$ to $15 \mathrm{MHz}$. The pellet samples were well polished to remove any roughness and the two surfaces of each pellet were coated with a silver paste as contact materials for electrical measurements. The DC electrical resistivity was carried out by using Keithley Electrometer and the AC resistivity and dielectric constant measurements as a function of frequency in the range $1 \mathrm{kHz}-10 \mathrm{MHz}$ at room temperature was carried out by using WAYNE KERR Impedance Analyzer (Model: 6500B).

\section{Results and Discussion}

\subsection{Structural properties}

Typical XRD patterns for the sample $\mathrm{Cu}_{1-\mathrm{x}} \mathrm{Mg}_{\mathrm{x}} \mathrm{Fe}_{2} \mathrm{O}_{4}$ with $x=0.4$ is illustrated in Fig. 1(a). The data showed intense sharp peaks and reveal well-crystalline single phase spinal structure for all $\mathrm{Mg}$ content. No other crystalline phase was observed indicating good stability of the ferrite compositions. The line profile, shown in Fig. was fitted for 7 peaks (220), (311), (222), (400), (422), (511) and (511).The existence of (311) peak, centre around $2 \theta \approx 35^{\circ}$ confirms the formation of cubic spinel structure. The well resolved peaks in the XRD pattern clearly indicate polycrystalline nature of the ferrites. The lattice constant for each peak of each sample was calculated by using the formula [7]: $a=d$ $\left(h^{2}+k^{2}+l^{2}\right)^{1 / 2}$ where $h, k, l$ are miller indices of the crystal planes. To determine the exact lattice parameter for each composition, Nelson-Riley method was used and the NelsonRiley function $F(\theta)$ is given as [8]: $F(\theta)=1 / 2\left[\cos ^{2} \theta / \sin \theta+\cos ^{2} \theta / \theta\right.$ ] where $\theta$ is the Bragg's angle. The values of lattice constant $\left(a_{o}\right)$ of all the peaks for each sample was plotted against $F(\theta)$ (not given). Then using a least square fit method exact lattice constant $\left(a_{\mathrm{o}}\right)$ 
was determined. The point where the least square fit straight line cut the $Y$-axis (where $F(\theta)=0)$ is the actual lattice parameter $(a)$ of the compositions.
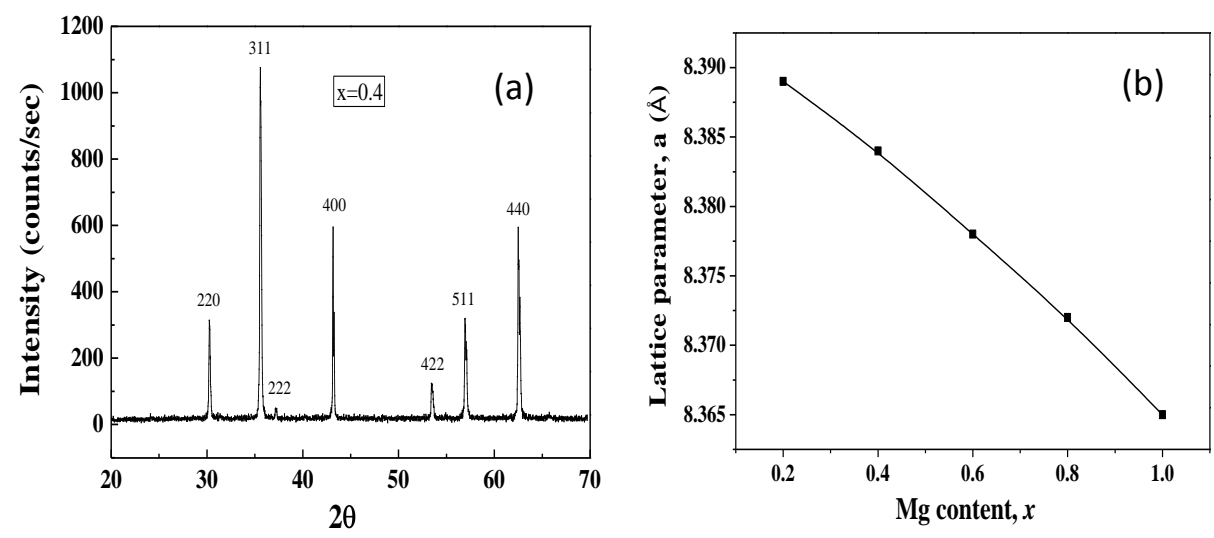

Fig.1. (a) Typical XRD pattern for $x=0.4$ of Cu-Mg ferrites (b) lattice parameter as a function of Mg content.

The lattice parameter $(a)$ as a function of $\mathrm{Mg}$ content $(x)$ is plotted in Fig. 1(b) which is evident that the lattice parameter $(a)$ is decreasing with the increase of $\mathrm{Mg}$ contents following Vegard's law [9]. This probably due to variation of ionic sizes of $\mathrm{Cu}^{2+}$ and $\mathrm{Mg}^{2+}$ ions since the ionic size of $\mathrm{Mg}^{2+}(0.66 \AA)$ is lower than that of of $\mathrm{Cu}^{2+}(0.73 \AA)$ [10]. This causes a decrease in size of the unit cell, where the length of the edge of unit cell decreases from 8.389 to $8.365 \AA$. The decrease of lattice parameter can be explained by the site occupancy. The tendency of $\mathrm{Cu}^{2+}$ cation to go to its preference site is consistent where their electron configuration has marked preference for the octahedral site. The divalent $\mathrm{Mg}^{2+}$ cations tend to occupy the tetrahedral site as this is favored by polarization effect of the oxygen atoms intermediate between A and B sites. This means that the tetrahedral sites are expended by an equal displacement of the four oxygen ions outwards along the body diagonal of the cube, at the same time the oxygen ions connected with octahedral sites move in such away as to shrink the size of the octahedral site by the same amount which leads to decrease of the lattice parameter values. The decrease in lattice parameter with increase in $\mathrm{Mg}$ content was also suggested the formation of a compositionally homogeneous solid state reaction. Similar decrease of lattice parameter has been observed by Pandit et. al. in Mg-Mn ferrites [11] and Bhosale in $\mathrm{Cu}-\mathrm{Mg}-\mathrm{Zn}$ ferrites [12].

\subsection{Microstructural analysis}

The grain size, grain boundary and porosity are important factors in microstructure, which influence the magnetic and electrical properties of $\mathrm{Cu}-\mathrm{Mg}$ ferrites. Fig. 2 shows Scanning Electron Microscope (SEM) micrographs for $\mathrm{Cu}_{1-\mathrm{x}} \mathrm{Mg}_{\mathrm{x}} \mathrm{Fe}_{2} \mathrm{O}_{4}$ ferrites with 
$x=0.2,0.4,0.6,0.8$ and 1.0. Fig. displays a homogeneous microstructure with small grain size distribution, approximately hexagonal grains (for $x=0.2,0.4,0.6$ ) and intergranular porosity. For $x=0.8$ and 1.0 grains are not clear compare to other samples as sintering temperature is low $\left(T_{\mathrm{s}}=1150^{\circ} \mathrm{C}\right)$. If sintering temperature greater than $1300^{\circ} \mathrm{C}$ then the grain will be visible, but for $\mathrm{Cu}$-contained composition cannot sinter more than $1225^{\circ} \mathrm{C}$ as melting temperature of $\mathrm{CuFe}_{2} \mathrm{O}_{4}$ is $1235^{\circ} \mathrm{C}$. Hexagonal grains are visible which indicating signs of the onset of intra-granular 'honeycombing'. The grain growth mechanism is compromised between driving force for grain boundary movement and retarding force of pores and inclusion during the sintering process.

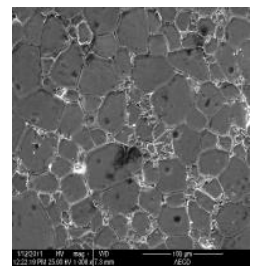

$x=0.2$

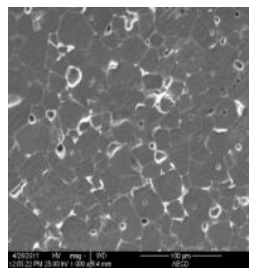

$x=0.4$

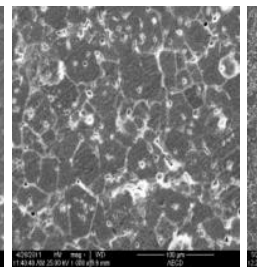

$x=0.6$

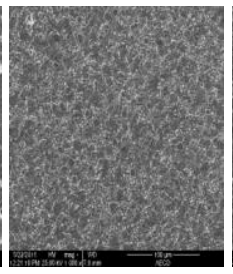

$x=0.8$

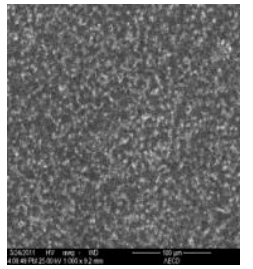

$x=1.0$

Fig. 2. SEM micrographs of $\mathrm{Cu}_{1-\mathrm{x}} \mathrm{Mg}_{\mathrm{x}} \mathrm{Fe}_{2} \mathrm{O}_{4}$ ferrites at $T_{\mathrm{s}}=1150^{\circ} \mathrm{C} / 2 \mathrm{~h}$.

The grain size was obtained by counting a sufficiently large number of grains and determined by linear intercept method from surface micrographs of samples. The average grain sizes of all samples are 34.36, 30.11, 24.83, 9.79 and $4.16 \mu \mathrm{m}$ for compositions $x=$ $0.2,0.4,0.6,0.8$ and 1.0 respectively indicating decrease in grain size with increasing $\mathrm{Mg}$ content. The decreasing trend in grain size was also observed in composition like $\mathrm{Ni}_{0.5} \mathrm{Mg}_{\mathrm{x}} \mathrm{Zn}_{05-\mathrm{x}} \mathrm{Fe}_{2} \mathrm{O}_{4}$ ferrites [13] and $\mathrm{Zn}_{1-\mathrm{x}} \mathrm{Mg}_{\mathrm{x}} \mathrm{Fe}_{2} \mathrm{O}_{4}$ ferrites [14] similar to present sample. This is because $\mathrm{MgO}$ acts as a microstructural stabilizator responsible for finer and uniform grain size [15]. Also $\mathrm{MgO}$ is a stable oxide avoids presence of divalent iron and there by circumvent tendency of discontinuous grain growth. Moreover the decrease of grain size could be due to increase of intra-granular porosity since the pores neutralize the driving force and the thickness of the grain boundary is found to increase with a decrease in grain size.

\subsection{Complex permeability}

Complex permeability is given by the complex notation $\mu=\mu^{\prime}-j \mu^{\prime \prime}$ where $\mu^{\prime}=$ real part of permeability (in phase), $\mu^{\prime \prime}=$ imaginary part of permeability (out of phase) and $\mathrm{j}=$ unit imaginary vector. The variation of complex permeability spectra for $\mathrm{Cu}_{1-\mathrm{x}} \mathrm{Mg}_{\mathrm{x}} \mathrm{Fe}_{2} \mathrm{O}_{4}$ ferrites samples sintered at $1150^{\circ} \mathrm{C}$ is shown in Fig. 3 which demonstrates that the real component of permeability, $\mu$ is reasonably constant with frequency but fall at higher frequencies. The imaginary component of permeability, $\mu$ " on the other hand, increases 
where as the real component $\mu$ falls to lower value of permeability. Similar type of behaviour of permeability has been observed in $\mathrm{Cu}-\mathrm{Zn}$ ferrites [16].

The general characteristic of the permeability spectra (Fig. 3) is that $\mu$ remains fairly constant in a certain frequency range, while at higher frequency it drops to lower value of permeability. The imaginary component $\mu^{\prime \prime}$ rises and increase quite abruptly making a peak at a certain frequency where the real component $\mu$ falls rapidly. The fairly constant $\mu$ values over a large frequency range show the compositional stability and quality of ferrites prepared by solid state reaction method. This is a desirable characteristic for various applications such as broadband pulse transformer and wide band read-write heads for video recording [17]. The flat region up to the frequency where it starts declining rapidly is known as the zone of utility of the ferrites. The frequency at which $\mu$ attains the highest value and after decreases, is known as the resonance frequency, $f_{\mathrm{r}}$ [18]. Resonance frequency, $f_{\mathrm{r}}$ is shifted to a higher value of frequency as increasing $\mathrm{Mg}$ content. However it was possible to observe the resonance peaks as $x=1.0$ seem to appear at frequencies higher than $15 \mathrm{MHz}$ which is maximum frequency limit used in present work. At sintering temp $1150^{\circ} \mathrm{C}, f_{\mathrm{r}}$ values increases from $1.8 \mathrm{MHz}$ to $10.3 \mathrm{MHz}$ from increasing $\mathrm{Mg}$ content where $\mu$ i decrease from 101 to 34, respectively.
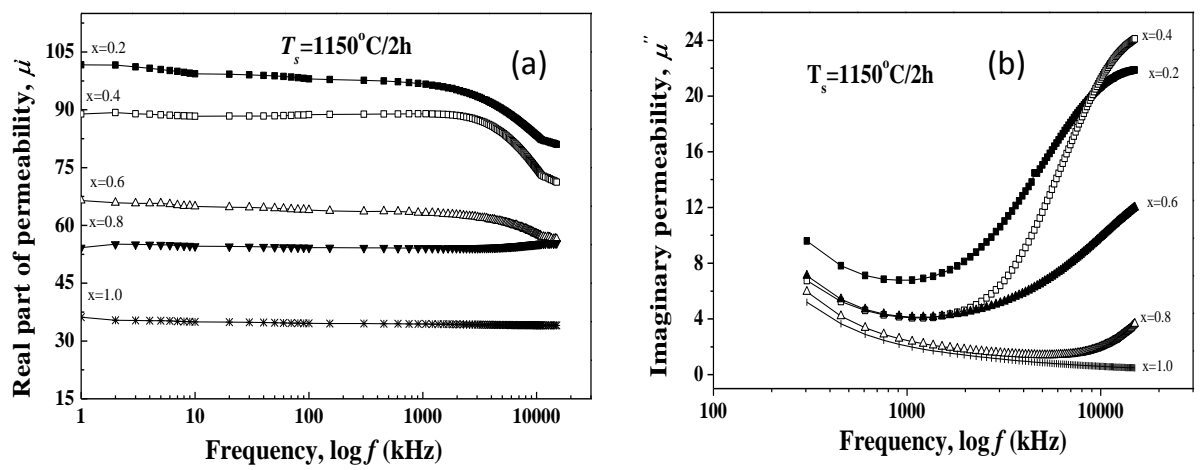

Fig. 3. (a) Real part and (b) imaginary part of permeability of $\mathrm{Cu}_{1-\mathrm{x}} \mathrm{Mg}_{\mathrm{x}} \mathrm{Fe}_{2} \mathrm{O}_{4}$ ferrites at $T_{\mathrm{s}}=1150^{\circ} \mathrm{C} / 2 \mathrm{~h}$.

In general, the complex permeability of polycrystalline ferrite is related to two different magnetization mechanisms: spin rotation and domain wall magnetizations [19]. At lower frequencies domain wall motion has major contribution to the permeability than spin rotation. The permeability due to domain wall motion is given by according Globus relation [20]:

$$
\mu_{i} \propto \frac{M_{s}^{2} D}{\sqrt{K_{1}}}
$$


where $D$ is the average grain size, $M_{\mathrm{s}}$ is the saturation magnetization and $K_{1}$ is the magneto-crystalline anisotropy constant. As the domain wall motion is greatly influenced by grain size, its contribution to the permeability is enhanced with increasing grain size. From Fig. 3 (a), it is observed that permeability decreases with increasing Mg content. This variation can be explained by considering variation of grain size and anisotropy constant with increased $\mathrm{Mg}$ substitution. As grain size decreases with $\mathrm{Mg}$ content so that initial permeability is also showing a decrease trend with increasing $\mathrm{Mg}$ content according to Globus relation. On the other hand, smaller grains are associated with more number of grain boundaries, which impede the domain wall motion leading to lower values of initial permeability [21]. Moreover, the microstructure exhibited increased homogeneity with finer grains; at the same time, there is an improved magnetocrystalline anisotropy constant, with the increase of $\mathrm{Mg}$ content. All these parameters put together, adversely affect the permeability, causing to decrease its value with increasing Mg content.

\section{4. $D C$ and $A C$ resistivity}

The variation of DC resistivity $(\log \rho)$ at room temp as a function of $\mathrm{Mg}$ concentration of $\mathrm{Cu}-\mathrm{Mg}$ ferrite is shown in Fig. 4 (a) in which resistivity decreases linearly with increasing $\mathrm{Mg}$ content. The observed variations in resistivity may be explained by Verwey's hopping mechanism [22]. According to Verwey the electronic conduction in ferrite is mainly due to hopping of electrons between ions of the same element present in more than one valance state, distributed randomly over crystallographically equivalent lattice sites. Ferrites structurally form cubic close packed oxygen lattices with cations at the octahedral (B) and the tetrahedral (A) sites. The distance between two metal ions at B sites is smaller than the distance between a metal ion at a B-site and another metal ion at an A-site. The electron hopping between $\mathrm{B}$ and $\mathrm{A}$ sites under normal conditions therefore has a very small probability compared with that for B-B hopping. Hopping between A and $\mathrm{B}$ sites does not exist for the simple reason that there are only $\mathrm{Fe}^{3+}$ ions and at the Asite and any $\mathrm{Fe}^{2+}$ ions formed during processing preferentially occupy B-site only. The hopping probability depends upon (1) the separation between the ions involved and (2) the activation energy. Moreover this decrease in DC resistivity may be attributed to changes in cation distribution formation excess $\mathrm{Fe}^{2+}$ ions and the difference in ionic size of the cations. Due to the smaller ionic size of $\mathrm{Mg}(0.66 \AA)$ as compared to $\mathrm{Cu}(0.73 \AA)$, its addition distorts the ferrites lattice. The distortions can affect the motion of changes causing a change in resistivity. 

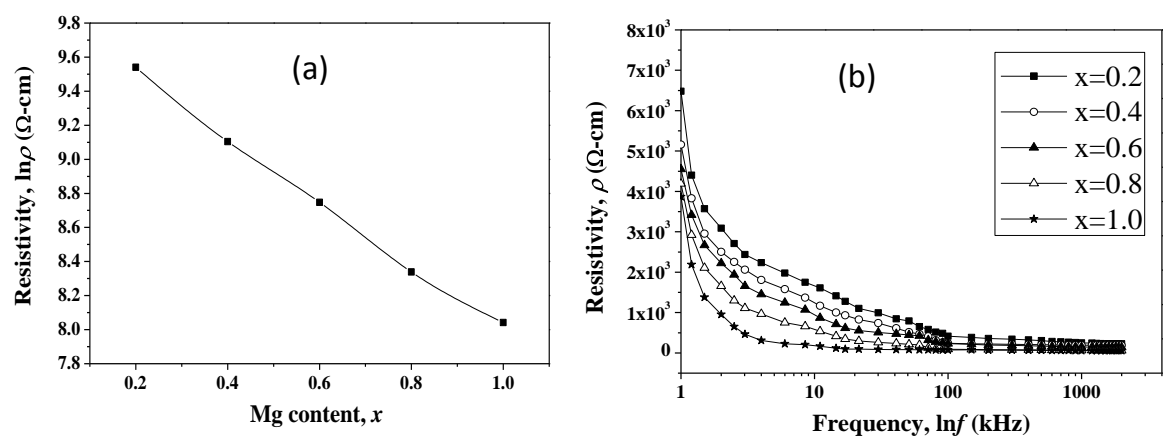

Fig. 4. Variation of resistivity with (a) Mg content, $x$ and (b) frequency.

The AC frequency decreases as the frequency increase from $1 \mathrm{KHz}$ to $2 \mathrm{MHz}$ and is illustrates in Fig. 4 (b). All the samples show the significant dispersion with frequency which is the normal ferrimagnetic behaviour. The resistivity of the ferrites is expected to decrease with an increase in the frequency; this may be due to the low dielectric constant and also depends on the porosity and compositions. This decrease in resistivity with increasing frequency can be explained by conduction mechanism of hopping charge carriers. The increase in frequency enhances the hopping frequency of charge carriers resulting in an increase in the conduction process thereby decreasing the resistivity. Ferrites are low mobility materials and the increase in conductivity does not mean that the number of charge carriers increases, but only the mobility of charge carriers increases. The minimum resistivity occurred when the frequency of the hopping charge carriers is equal to the applied field frequency termed as resonance frequency i.e. the jumping frequency of hopping charge carriers are almost equal to that the applied ac dielectric field. A low resistivity is found to be associated in these ferrites with a high dielectric constant. The resistivity is mainly governed by charge carriers mobility rather than carriers concentration. The resistivity is found to decrease with frequency, which agrees with the relation [23]:

$$
\rho=\frac{1}{f \varepsilon_{0} \varepsilon^{\prime \prime}}=\frac{1}{f \mathcal{E}_{0} \varepsilon^{\prime} \varepsilon^{\prime \prime} \tan \delta}
$$

where $\varepsilon_{o}$ is the vacuum permittivity, $\varepsilon^{\prime}$ and $\varepsilon^{\prime \prime}$ are real and imaginary part of dielectric constant and $\tan \delta$ is the tangent of dielectric loss.

\subsection{Dielectric constant}

The effect of $\mathrm{Mg}^{2+}$ content substitution on dielectric constant, $\varepsilon$ at different frequency is presented in Fig. 5 (a) in which a decrease of $\varepsilon$ is observed with successive substitution of $\mathrm{Mg}$ content in $\mathrm{Cu}-\mathrm{Mg}$ ferrite. It was reported that $\mathrm{Cu}^{2+}$ ion strongly prefers the 
occupation of tetrahedral (A site) while $\mathrm{Mg}^{2+}$ ion and $\mathrm{Fe}$ ion partially occupy A-site and

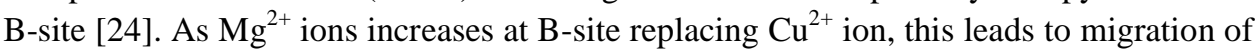
some $\mathrm{Fe}$ ions from $\mathrm{B}$-site to $\mathrm{A}$-site. As a result, the number of $\mathrm{Fe}^{2+}$ and $\mathrm{Fe}^{3+}$ and at $\mathrm{B}$-site decrease between which the electron exchange interaction takes place and is responsible for electric conduction and dielectric polarization [25]. The hopping of localized electrons at the $d$-shell for $\mathrm{Fe}^{2+}$ and $\mathrm{Fe}^{3+}$ ions causes the dielectric polarization. The mechanism of electric conduction in ferrites is similar to that for dielectric polarization. Therefore, the marked decrease in the dielectric constants takes place as $\mathrm{Mg}^{2+}$ ions substitution increase.
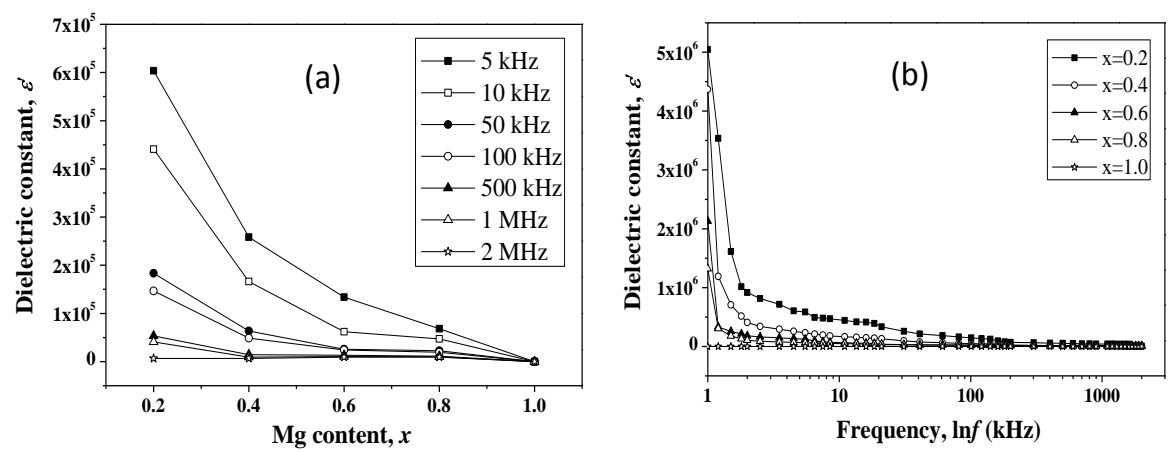

Fig. 5. Variation of dielectric constant with (a) Mg content, $x$ and (b) frequency.

Fig. 5 (b) shows the variation of dielectric constant with frequency from $1 \mathrm{kHz}$ to 2 $\mathrm{MHz}$ in which the dielectric constant decreases with increasing frequency. This is normal behaviour observed in most of the ferromagnetic materials, which may be due to the interfacial polarization as predicted by Maxwell-Wagner [26]. According to their model, the dielectric structure of a ferrite material is assumed to be made up of two layers. First layer being a conducting layer consists of large ferrite grains and the other being the grain boundaries that are poor conductors. According to Rabinkin [27] the polarization in ferrites is through a mechanism similar to the conduction process. By electron exchange between $\mathrm{Fe}^{2+}$ and $\mathrm{Fe}^{3+}$, the local displacement of electrons in the direction of the applied field occurs and these electrons determine the polarization. The polarization decreases with increasing frequency and then reaches a constant value due to the fact that beyond a certain frequency of external field, the electron exchange between $\mathrm{Fe}^{2+}$ and $\mathrm{Fe}^{3+}$ cannot follow the alternating field. The large value of $\varepsilon^{\prime}$ at lower frequency is due to the predominance of species like $\mathrm{Fe}^{2+}$ ions, interfacial dislocations pile ups, oxygen vacancies, grain boundary defects etc. [28], while the decrease in $\varepsilon$ with frequency is natural because of the fact that any species contributing to polarizability is found to show lagging behind the applied field at higher and higher frequencies. 


\section{Conclusions}

$\mathrm{Cu}_{1-\mathrm{x}} \mathrm{Mg}_{\mathrm{x}} \mathrm{Fe}_{2} \mathrm{O}_{4}$ ferrites prepared through solid state reaction showed single phase spinel structure with no secondary phases. By increasing the $\mathrm{Mg}$ content the lattice parameters decreased as a result of the smaller ionic radius of $\mathrm{Mg}^{2+}$ as compared to $\mathrm{Cu}^{2+}$ ion. The grain size is found to decrease with increasing $\mathrm{Mg}$ content. The initial permeability is found to be constant up to certain frequency range showing compositional stability and quality of materials which is applicable for Multilayer Chip Inductors (MLCI). The decrease of the initial permeability is observed with the increase of $\mathrm{Mg}$ content due to a decrease of grain size and to variation of magneto-crystalline anisotropy constant. The decrease in DC resistivity with increasing $\mathrm{Mg}$ content is attributed to change in cation distribution, formation of excess $\mathrm{Fe}^{2+}$ ions and the difference in ionic size of the cation. $\mathrm{AC}$ resistivity decreases with increasing frequency due to the conduction mechanism of hopping charge carriers. The dielectric constant decreases with increasing $\mathrm{Mg}$ content as well as with increasing frequency which has been explained on the basis of space charge polarization resulting from electron displacement which is due to a major contribution to the dielectric constant in ferrites. Bottom up technique will be used in future to see the particle size effects on the properties of these ferrites.

\section{Acknowledgements}

The author (Shahida Akhter) is grateful to the 'Bangabandhu Fellowship on Science, Information and Technology' fund of the People's Republic of Bangladesh for granting her a Ph.D. fellowship for carrying out this work. She is also grateful to Materials Science Division of Atomic Energy Centre, Dhaka (AECD) for allowing her prepare the samples and to utilize its laboratory facilities.

\section{References}

1. M. Sugimoto, J. Am. Ceram. Soc. 82, 269 (1999). http://dx.doi.org/10.1111/j.1551-2916.1999.tb20058.x

2. S. C. Watawe, U. A. Bamne, S. P. Gonbare, and R. B. Tangsali, Mater. Chem. Phys. 103, 323 (2007). http://dx.doi.org/10.1016/j.matchemphys.2007.02.037

3. J. Darul and W. Nowicki, Radiat. Phys. Chem. 78, S109 (2009). http://dx.doi.org/10.1016/j.radphyschem.2009.02.009

4. Q. Chen and Z. J. Zhang, Appl. Phys. Lett. 71, 3156 (1998). http://dx.doi.org/10.1063/1.122704

5. F. A. Benko and E. P. Koffyberg, Mater. Res. Bull. 21, 1183 (1986). http://dx.doi.org/10.1016/0025-5408(86)90045-0

6. Z. Tinashu, P. Hing, Z. Jianeheng, and K. Kingbing, Mater. Chem. Phys. 61, 192 (1999). http://dx.doi.org/10.1016/S0254-0584(99)00133-9

7. B. D. Cullity, 'Elements of X-ray diffraction’ (Addison-Wesely, London, 1959) p. 355.

8. J. B. Nelson and D. P. Riley, Proc. Phys. Soc. (Lond) 57, 160 (1945). http://dx.doi.org/10.1088/0959-5309/57/3/302

9. L. Vegard, Physica 5, 17 (1921).

10. D. N. Bhosale, S. R. Sawant, S. A. Gangal, R. R. Mahajan, and P. P. Bakare, Mater. Sci. Eng. B, 65, 79 (1999). http://dx.doi.org/10.1016/S0921-5107(99)00182-8 
11. A. A. Pandit, A. R. Shitre, D. R. Shenguli, and K. M. Jadhah, Mater.Sci. 40, 423 (2005). http://dx.doi.org/10.1007/s10853-005-6099-x

12. D. N. Bhosale, N. D. Choudhari, S. R. Sawant, and P. P. Bakare, J. Magn. Magn. Magn. 173, 51 (1997). http://dx.doi.org/10.1016/S0304-8853(97)00178-9

13. A. K. M. A. Hossain, T. S. Biswas, T. Yanagida, H. Tanaka, H. Tabata, and T. Kawai, Mater. Chem. Phys. 120, 461 (2010). http://dx.doi.org/10.1016/j.matchemphys.2009.11.040

14. A. Daigle, J. Modest, A. L. Geiler, S. Gillette, Y. Chen, M. Geiler, B. Hu, S. Kim, K. Stopher, C. Vittoria, and V. G. Harris, Nanotechn. 22, 305708 (2011). http://dx.doi.org/10.1088/0957-4484/22/30/305708

15. E. Rezlescu, N. Reslescu, P. D. Popa, L. Reslescu, C. Pasnicu, and M. I. Craus, Mater. Res. Bull. 33(6), 915 (1998). http://dx.doi.org/10.1016/S0025-5408(98)00050-6

16. Shahida Akhter, D. P. Paul, M. A. Hakim, S. Akhter, D. K. Saha, B. Anjuman, and F. Islam, J. Sci. Res. 4 (3), 551 (2012).

17. T. Tsutaoka, J. Appl. Phys. 33, 2789 (2003). http://dx.doi.org/10.1063/1.1542651

18. R. Valenzuela, 'Magnetic Ceramies' (Cambridge University Press, Cambridge, 1994). http://dx.doi.org/10.1017/CBO9780511600296

19. T. Nakamura, J. Magn. Magn. Magn. 168, 285 (1997). http://dx.doi.org/10.1016/S0304-8853(96)00709-3

20. A. Globus, P. Duplex, M. Guyot, IEEE Trans. Magn. 7, 617 (1971). http://dx.doi.org/10.1109/TMAG.1971.1067200

21. A. Goldman, Modern Ferrite Technology, $2^{\text {nd }}$ Ed. (Springer Publishing, 2006).

22. E. J. W. Verwey, J. H. Boer, Rec. Trans. Chem. Phys., 55, 531 (1936). http://dx.doi.org/10.1002/recl.19360550608

23. M. A. Ahmed, E. Ateia, S.I. El Dek, Material Lett. 57, 4256 (2003). http://dx.doi.org/10.1016/S0167-577X(03)00300-8

24. B. Bhise and M. Dongar, S. patil, S. Sawant, J. Mater. Sci. Lett. 10, 922 (1991). http://dx.doi.org/10.1007/BF00724783

25. P. Reddy, T. Rao, and S. Rao, J. Less Common. Met. 79, 1 (1981). http://dx.doi.org/10.1016/0022-5088(81)90045-X

26. J. C. Maxwell, 'Electric and Magnetism' (Oxford University Press, New York, 1973) pp. 828.

27. L. Rabin and Z. Novikova, 'Ferrites' Izd. Nauk. Tekhn. Minsik, USSR, 146 (1969).

28. J. Zhu, K. J. Tseng, and C. F. Foo, IEEE Trans. Magn. 36, 3408 (2000). http://dx.doi.org/10.1109/20.908842 